\title{
THE DRIFTLESS GAS SCINTILLATION PROPORTIONAL COUNTER
}

\author{
M. Heppener, H. J. M. Aarts and D. G. Simons \\ Laboratory for Space Research Leiden, Niels Bohrweg 2, 2333 CA Leiden, \\ The Netherlands
}

\begin{abstract}
In this article we present the performance characteristics of a so-called driftless Gas Scintillation Proportional Counter which operates in the energy range $0.1-15 \mathrm{keV}$. Typically, an energy resolution of $30.8 \%$ Full Width at Half Maximum (FWHM) is obtained at $0.28 \mathrm{keV}$ (C-K radiation) whereas a rejection efficiency of $99.6 \%$ for Co-60 induced background events is achieved in the energy range $0.4-10 \mathrm{keV}$. By making use of a position sensitive microchannelplate read-out a typical position resolution of $0.95 \mathrm{~mm}$ FWHM at $7.5 \mathrm{keV}$ (Ni-K radiation) is obtained.

\section{INTRODUCTION}

The driftless Gas Scintillation Proportional Counter (GSPC) differs from a conventional one in the absence of a separate absorption region. In stead, the X-ray photons are absorbed directly in the scintillation region, in which a high electric field is present. The advantages of the driftless concept are an expected better low energy performance due to the absence of electron loss to the detector entrance window and an improved background rejection capability as a result of the smaller electron cloud size. By making use of an Csl-coated microchannelplate (MCP) with a wedge and strip anode as detector for the scintillation light the GSPC can be made position sensitive. In this way an instrument is obtained that is very well suited for a variety of imaging $X$-ray applications including the use as focal plane instrument for grazing incidence telescopes such as employed in X-ray astronomy.
\end{abstract}

\section{EXPERIMENTAL ARRANGEMENT}

\section{Description of the detector and principle of operation}

The driftless GSPC consisted of a cylindrical Macor body, $30 \mathrm{~mm}$ deep and with an internal diameter of $60 \mathrm{~mm}$. The $25 \mathrm{~mm}$ diameter entrance window was made of $3.3 \mu \mathrm{m}$ thick polypropylene coated with colloidal carbon to provide the electrical conductivity needed to apply the high voltage. In order to withstand the $1 \mathrm{~atm}$. differential pressure across the thin window a support grid has been included. The exit window of the gascell was made of $4 \mathrm{~mm}$ thick $\mathrm{MgF}_{2}$ with an aperture of $40 \mathrm{~mm}$. A grounded grid of 80 $\%$ optical transmission was mounted just above the exit window in order to define the electric field. As scintillation gas pure xenon at a pressure of $1 \mathrm{~atm}$. was used. The maximum scintillation voltage during the experiments was $11.7 \mathrm{kV}$. This corresponds to a reduced electric field of ca. $5 \mathrm{~V} \mathrm{~cm} \mathrm{corr}^{-1}$, well below the onset of the gas amplification process (ca. $6 \mathrm{~V} \mathrm{~cm}{ }^{-1}$ Torr $^{-1}$ ) $/ 1 /$. A SAES getter at an operating temperature of $400^{8} \mathrm{C}$ was used to continuously purify the noble gas.

After absorption of an X-ray photon in the xenon gas a cloud of primary electrons is produced which is accelerated instantaneously by the electric field. During acceleration the electrons acquire sufficient energy to create xenon excimers which deexcite under the emission of UV photons in the waveband 150$190 \mathrm{~nm} / 2$. The number of these photons is proportional to the number of primary electrons and hence to the energy of the incident X-ray photon. In a driftless GSPC the light output is also dependent on the absorption depth of the incoming X-ray photon. Therefore the duration of scintillation (hereafter called burstlength) of each event has to be measured and the pulse height corrected accordingly. Detection of the UV photons was normally accomplished by means of a $3.5^{\prime \prime}$ EMI photomultiplier tube (PMT) with a quartz window and a bialkali photocathode (type D $319 \mathrm{QB}$ ) with an effective quantumefficiency (QE) of approximately $20 \%$.

For position sensitive measurements a $39 \mathrm{~mm}$ diameter microchannelplate chevron, coated with 1400 $\mathrm{nm}$ Csl, was used as read-out device. The quantumefficiency of the CsI photocathode has been measured to be $9 \%$ at an incident angle of $20^{\circ}$. Details on the fabrication and calibration as well as the dependence of the quantumefficiency on wavelength and angle of incidence (in the ranges $25-200 \mathrm{~nm}$ and $0-90^{\circ}$, respectively) of this device can be found in ref./3/. The spatial distribution of the electron cloud leaving the MCP stack is determined by means of a wedge and strip anode/4/. This device has the advantage of yielding a good linearity while needing only three signal connections. The incoming 
position of the X-ray photon is then calculated as the centre of gravity of this distribution. in order to prevent degradation of the photocathode the whole assembly has been stored under vacuum continuously. The LiF entrance window of the MCP housing served at the same time as exit window for the GSPC.

\section{Sianal processing}

Several parameters have to be extracted from the output of the read-out device. These are: pulse height pulse shape and, optionally, position. Pulse shape information is needed for two reasons. First, as already mentioned above, the burstlength has to be measured in order to correct for pulse height variations arising from the variation in scintillation pathlengths. Second, a very powerful background rejection technique is based on the different pulse shapes obtained from (point-like) X-ray events when compared to those from extended tracks originating from the passage of high-energy particles through the detector volume. It will be shown that especially the pulse decay time is an extremely sensitive parameter in this respect.

A detailed description of the analogue and digital signal processing equipment can be found in refs. $/ 5 /$ and $/ 6 /$. Briefly, the pulse height signal was obtained by shaping the output of the charge sensitive preamplifier coupled to the read-out system. Burstlength and decay time information was derived from the differentiated preamplifier signal (i.e. the scintillation time profile, see fig.8) by means of constant fraction timing. Finally, in the case of the MCP read-out three preamplifiers were used, each for one segment of the wedge and strip anode, and shaped independently. Burstlength and pulse height were determined in the same way as for the EMI PMT.

Storage of the data as well as subsequent data analysis was performed by the AT running programs written in the scientific computer language ASYST. $X$ and $Y$ positions were calculated as the fractions $f_{s}$ and $f_{w}$ of the total charge falling on the $s$ and $w$ anodes, respectively, normalized to the total amount of charge leaving the MCP, according to:

$$
\begin{aligned}
& f_{s}=Q_{s} /\left(Q_{s}+Q_{w}+Q_{z}\right) \\
& f_{w}=Q_{w} /\left(Q_{s}+Q_{w}+Q_{z}\right)
\end{aligned}
$$

with $Q_{s}, Q_{w}$ and $Q$ the charge signals on the $s, w$ and $z$ anodes, respectively. The total pulse height was in this case calculated as the sum of the s-, $w$ - and $z$-signals. The analysis programs accounted also for burstlength correction of the raw pulse height spectra, the generation of histograms and application of Gauss fits to the pulse height and position spectra to obtain energy and position resolutions.

\section{X-ray generation}

$\mathrm{X}$-ray photons with an energy up to $2 \mathrm{keV}$ (i.e. B-K, C-K and Al-K radiation) were produced in an X-ray generator comprising an electron emitting filament and a cathode coated with the relevant material. If necessary a filter was used to suppress the bremsstrahlung continuum. For the higher energies (Ti-K, $\mathrm{Cr}-\mathrm{K}$ and $\mathrm{Fe}-\mathrm{K}$ ) fluorescent $\mathrm{X}$-ray photons were produced from a suitable target, excited with characteristic radiation and bremsstrahlung from a Ni anode. Care has been taken that, whenever appropriate, the beam spot size was at least a factor two smaller than the FWHM position resolution of the GSPC.

\section{RESULTS}

\section{Eneray resolution}

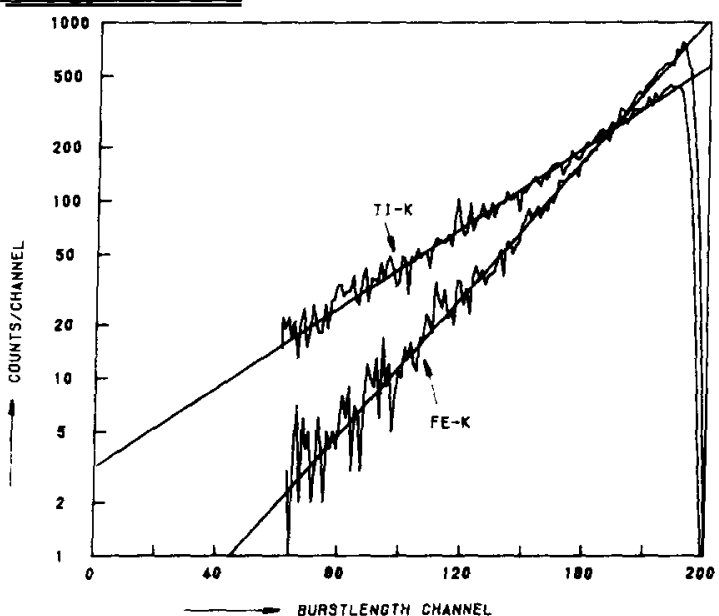

Fig.1. Measured burstlength distributions for TI-K and Fe$K$. The solid lines represent the exponential fits to the data.

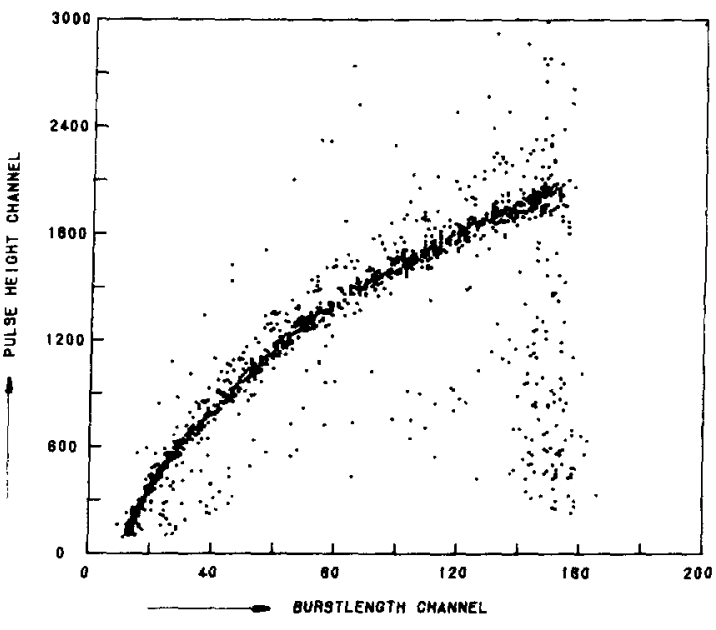

Fig.2. The measured burstlength/pulse height response for $\mathrm{Ag}-\mathrm{K} \alpha$ and $\mathrm{Ag}-\mathrm{K} \theta$ X-rays. 
Since the QE of the EMI photomultiplier was approximately twice as high as that of the CsI-coated MCP we will present in this section only results obtained with the former read-out device.

Fig. 1 shows the measured burstlength distributions for Ti-K and Fe-K X-rays (4.5 and $6.4 \mathrm{keV}$, respectively) obtained from the differentiated preamplifier signal. From the exponential fits to the data one obtains mean absorption depths of 6.1 and $3.5 \mathrm{~mm}$, respectively, which is in good agreement with the attenuation cross sections for $\mathrm{Xe}$ as reported by Veigele/7/. As a result of these non-negligible absorption depths the pulse height distributions show an appreciable tail towards the lower energies. In fig. 2 the correlation between pulse height and burstlength is shown. Restored pulse height spectra are obtained by applying a burstlength correction algorithm based on a second order polynomial fit to the data in fig.2. In fig. 3 the results before and after correction are shown for Ti-K X-rays. The energy resolution derived from a Gauss fit to the data (taking into account the presence of the $\mathrm{K} \beta$ line at $4.9 \mathrm{keV}$ ) is $8.5 \%$. In the same way an energy resolution of $7.3 \%$ is obtained at $\mathrm{Fe}-\mathrm{K}_{\alpha}(6.4 \mathrm{keV})$. By irradiating the GSPC with a radioactive ${ }^{109} \mathrm{Cd}$ source the energy resolution at $22.1 \mathrm{keV}\left(\mathrm{Ag}-\mathrm{K}_{\alpha}\right)$ has been measured to be $4.2 \%$ (fig.4)
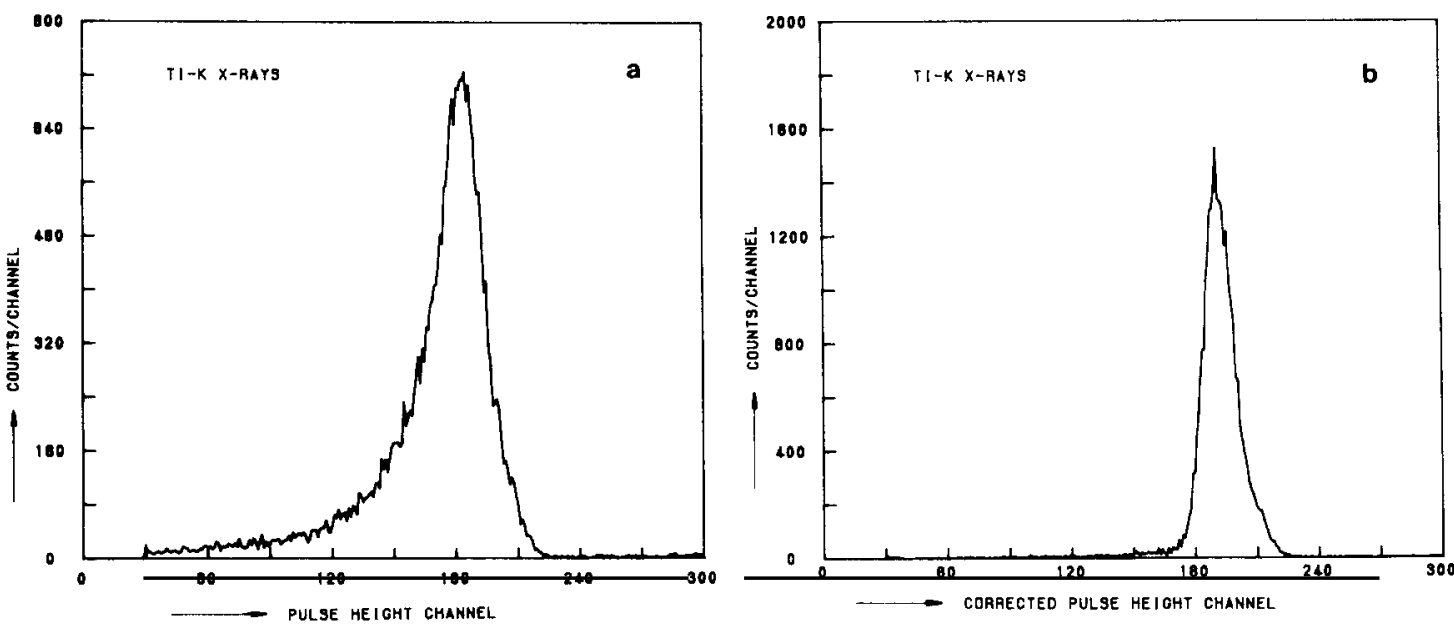

Fig.3. The raw (a) and burstlength corrected (b) pulse height distribution measured for Ti-KX-rays.

For the lower energies the influence of differences in scintillation pathlengths is negligible as a result of the small absorption depths in xenon. The measured energy resolutions at Al-K $(1.48 \mathrm{keV})$ and $\mathrm{C}-\mathrm{K}(0.28$ $\mathrm{keV}$ ) are 14.7 and $30.8 \%$ FWHM, respectively. In fig.5 the observed pulse height distribution obtained with an anode coated with a carbon-boron mixture is shown. Fitting the data with two gaussians, the parameters of one of which correspond to those obtained for the pure carbon spectrum, an energy resolution of $36.4 \%$ at $0.18 \mathrm{keV}$ is obtained.

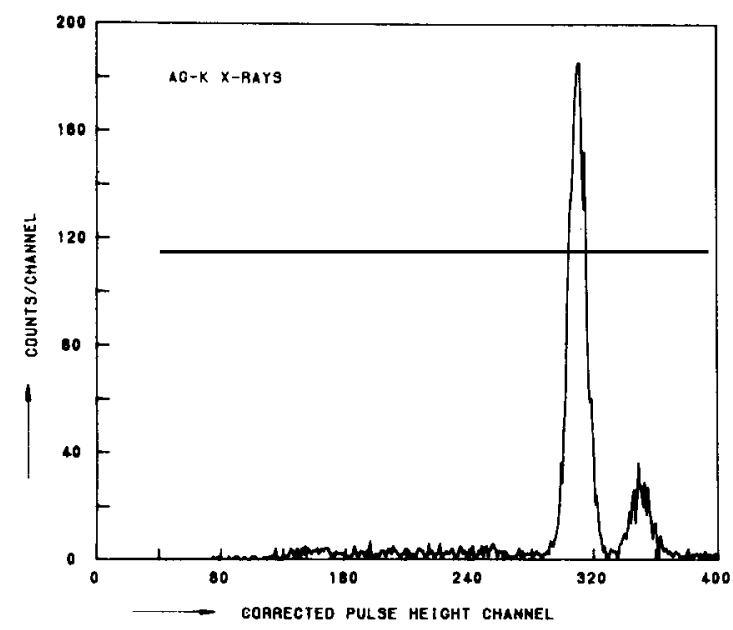

Fig.4. Corrected pulse height distribution for Ag-K X-rays.

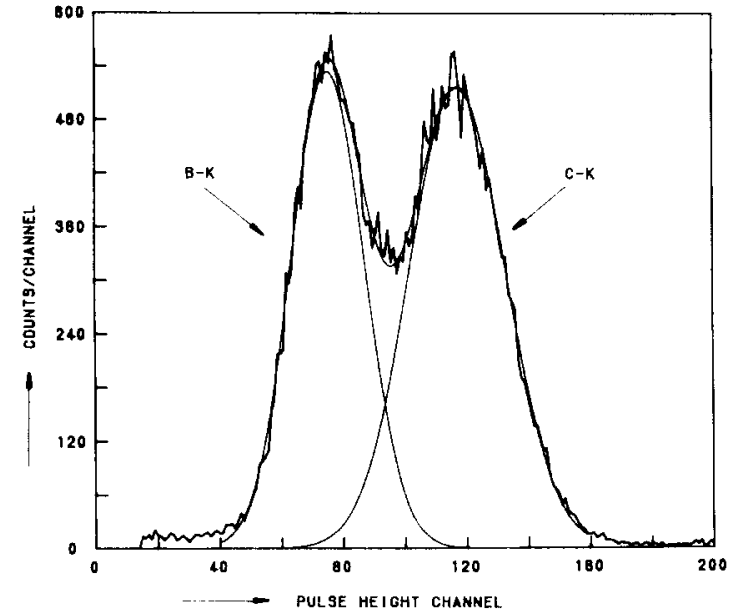

Fig.5. The observed pulse height distribution obtained from a mixed boron-carbon target. The solid lines result from a gaussian fit to the data performed to obtain the width of the boron peak. 


\section{Position resolution}

Position resolution measurements were necessarily only performed with the MCP read-out since the EMI PMT does not give spatial information.

Fig.6 shows the theoretical and experimental values of the FWHM position resolution as measured at various $X$-ray energies. In this figure also the contribution of lateral diffusion of the primary electron cloud has been included, both for a standard and a driftless configuration. As can be seen the position resolution is completely determined by the QE of the MCP. The advantage of the driftless concept will however become appreciable when using a read-out system with a QE comparable to that of a conventional PMT.

A particularly interesting feature is the dependence of the measured position on the absorption depth for off-axis X-rays. This phenomenon, which is due to the finite size of the read-out system, can be seen most clearly in fig.7 where the measured mean positions at different absorption depths for Ti-K X-rays are depicted. It can be shown that the measured radial position $\mathrm{A}_{\mathrm{m}}$, relative to the centre of the detector obeys the following simple relationship:

$$
R_{m}(L)=R_{0} /(1+c . L)
$$

with $L$ the scintillation pathlength, $R_{0}$ the incoming position and $c$ a constant equal to $0.020 \mathrm{~mm}^{-1}$. For very large read-out systems $c$ should approach zero. Eq.2 provides a simple correction procedure for the reconstruction of the incoming position for off-axis photons.

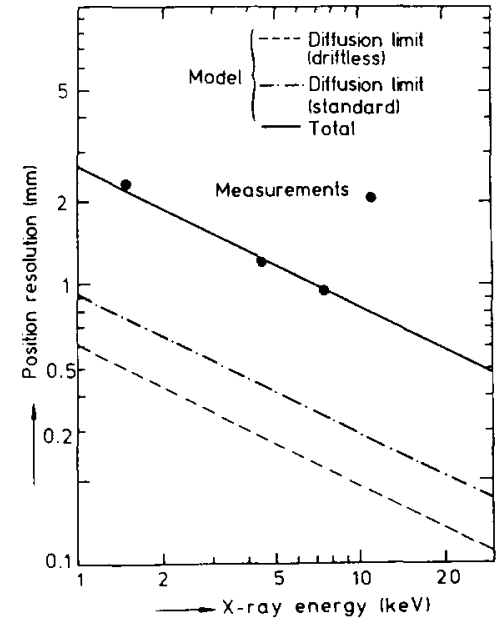

Fig.6. Measured and calculated FWHM position resolutions as a function of X-ray energy. Also indicated are the diffusion limits to the position resolution for a driftless and a standard GSPC. dent on the detector at different positions at three absorption depths: $0 \mathrm{~mm}$ (circles), $5 \mathrm{~mm}$ (triangles) and $10 \mathrm{~mm}$ (squares).

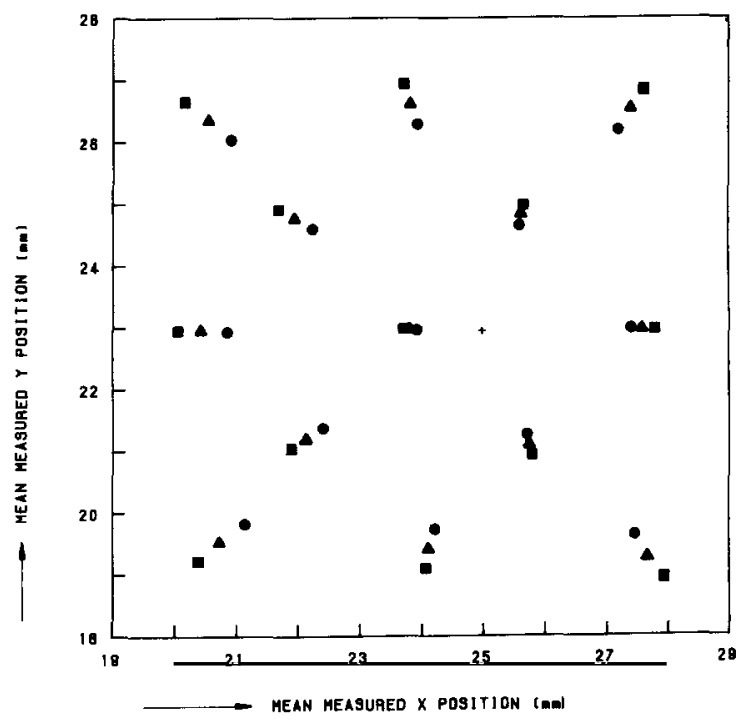

\section{Background rejection}

Background events were simulated by placing $a^{60} \mathrm{Co}$ source at the side of the detector. The issuing gamma ray photons in the $\mathrm{MeV}$ range liberate energetic electrons from the detector walls and in the counter gas via the Compton effect. These electrons create extended tracks of ionization in the gas. True $X$-ray events, on the other hand, have a very limited extent which is determined by the diffusion of the primary electron cloud only. This difference can be exploited as background rejection mechanism by measuring the decay time of the light pulse for each event. Typical X-ray and background pulse shapes can be seen in fig.8. With the exception of ionization tracks that are exactly parallel to the exit window one expects the decay time of background events to be always longer than for $X$-ray events. This can be seen in fig. 9 where the sharp decay time distribution obtained with $5.9 \mathrm{keV} X$-rays from an ${ }^{55} \mathrm{Fe}$ source is compared to the distribution obtained with the ${ }^{60} \mathrm{Co}$ source. Since the lower $1 \mathrm{~cm}$ of the detector does not contribute significantly to the sensitivity of the instrument in the desired energy band the distribution for background events compatible with X-ray absorption in the upper $2 \mathrm{~cm}$ of the detector is also included. By applying a suitable decay time window simultaneously with the burstlength window needed to reject events from the lower $1 \mathrm{~cm}$ of the detector a rejection efficiency of $98.9 \%$ is obtained at an X-ray acceptance level of $85 \%$. Off-line application of an absorption depth-dependent burstlength window can 
improve the rejection efficiency even further to $99.6 \%$ in the energy range $0.4-10 \mathrm{keV} / 6 /$. Additional improvements are expected from the use of the spatial information provided by the read-out system.
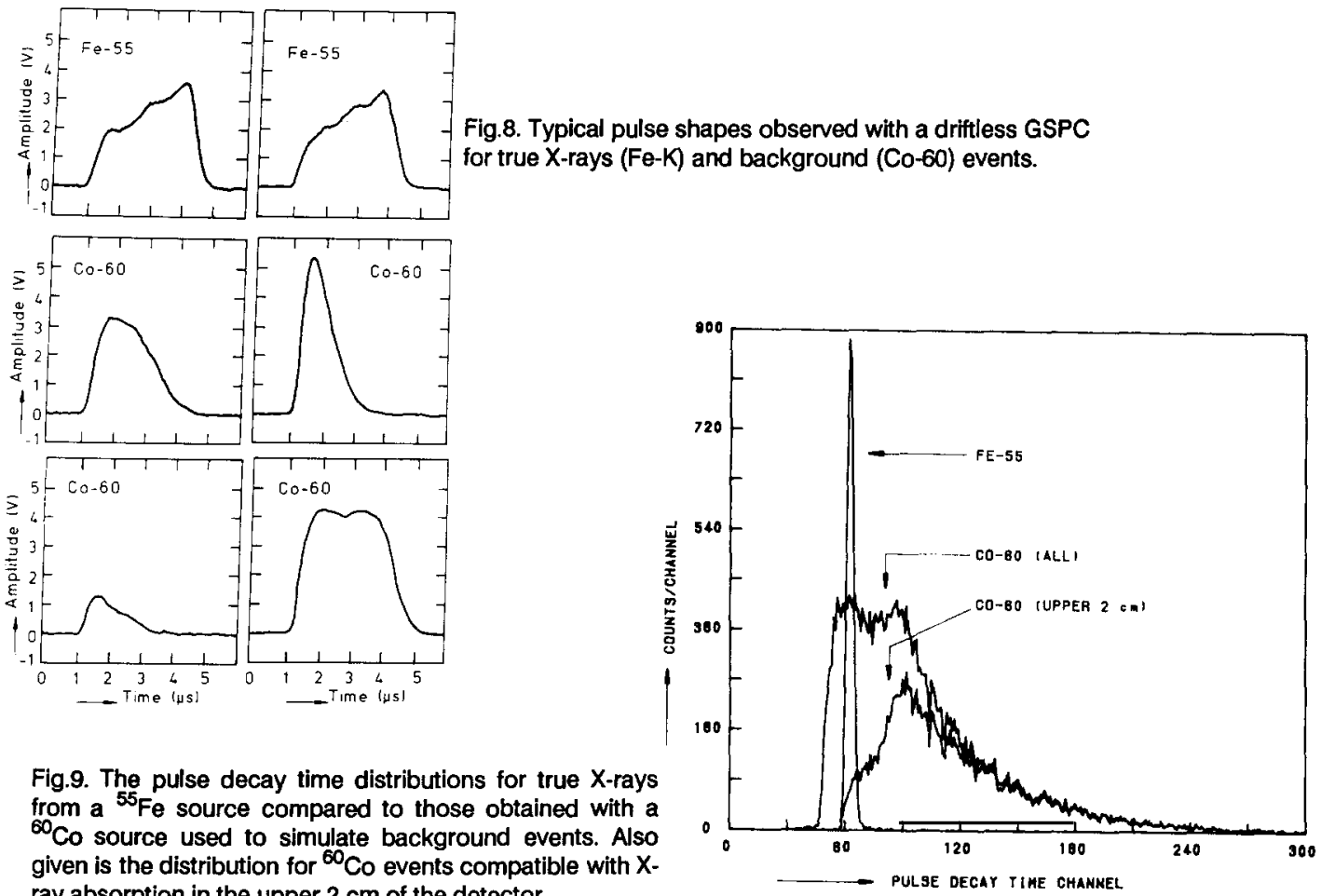

Fig.9. The pulse decay time distributions for true $X$-rays from a ${ }^{55} \mathrm{Fe}$ source compared to those obtained with a ${ }^{60} \mathrm{Co}$ source used to simulate background events. Also given is the distribution for ${ }^{60}$ Co events compatible with $X$ ray absorption in the upper $2 \mathrm{~cm}$ of the detector.

\section{CONCLUSIONS}

Driftless gas scintillation proportional counters provide superior performance in terms of energy resolution and background rejection when compared to GSPC's with a separate drift region. Most striking in this respect are the energy resolutions obtained at sub-keV X-ray energies such as B-K or C-K radiation. From these results it is clear that the problem of primary electron loss to the detector entrance window $/ 8$ / has been solved. The background rejection efficiencies reported here surpass those obtained with gas proportional counters in a comparable energy range/9/. The position resolutions of our instrument are completely determined by the QE of the MCP read-out. It appears to be difficult to coat the MCP with other materials exhibiting higher quantumefficiencies at $170 \mathrm{~nm}$ such as $\mathrm{Cs}_{2} \mathrm{Te}$. Moreover, the high resistivity of the MCP material gives rise to gain degradation at higher countrates $/ 10 /$. Further the expected lifetime of channelplates is not compatible with the use in a long duration satellite mission. Therefore the solution of this problem is sought in the use of a position sensitive PMT that has been developed recently $/ 11 /$. Results of this investigation will be published shortly.

\section{ACKNOWLEDGEMENTS}

The authors would like to thank A. van der Heijden, L. de Jong, M.G.A. Kengen and L.Weijts for continuous support during the measurements. The Laboratory for Space Research Leiden is supported financially by the Netherlands Foundation for Scientific Research (NWO).

\section{REFERENCES}

1. T.H.V.T. Dias, A.D. Stauffer and C.A.N. Conde, IEEE Trans. Nucl. Sci. NS-34 (1983) 389.

2. A. Gedanken, J. Jortner, B. Raz and S. Szöke, J. Chem. Phys. 57 (1972) 3456.

3. D.G. Simons, G.W. Fraser, P.A.J. de Korte, J.F. Pearson and L. de Jong, Nucl. Instr. and Meth. A261

(1987) 579.

4. O.H.W. Siegmund, R.F. Malina, K. Coburn and D. Werthimer, IEEE Trans. NuCl. Sci. NS-31 (1984) 776.

5. D.G. Simons, Thesis, Leiden University, 1988.

6. D.G. Simons and P.A.J. de Korte, accepted for publication in Nucl. Instr. and Meth. A.

7. Wm.J. Veigele, Atomic data tables 5 (1973) 51.

8. H. Inoue, K. Koyama, M. Matsuoka, Y. Tanaka and H.Tsunemi, Nucl. Instr. and Meth. A157 (1978)

295.

9. T.A. Bailey, A. Smith and M.J.L. Turner, NuCl. Instr. and Meth. 155 (1978) 177.

10. D.G. Simons, P.A.J. de Korte and M. Heppener, NuCl. Instr. and Meth. A273 (1988) 512.

11. H. Kume, S. Muramatsu and M.lida, IEEE Trans. Nucl. Sci. NS-33 (1986) 359. 